
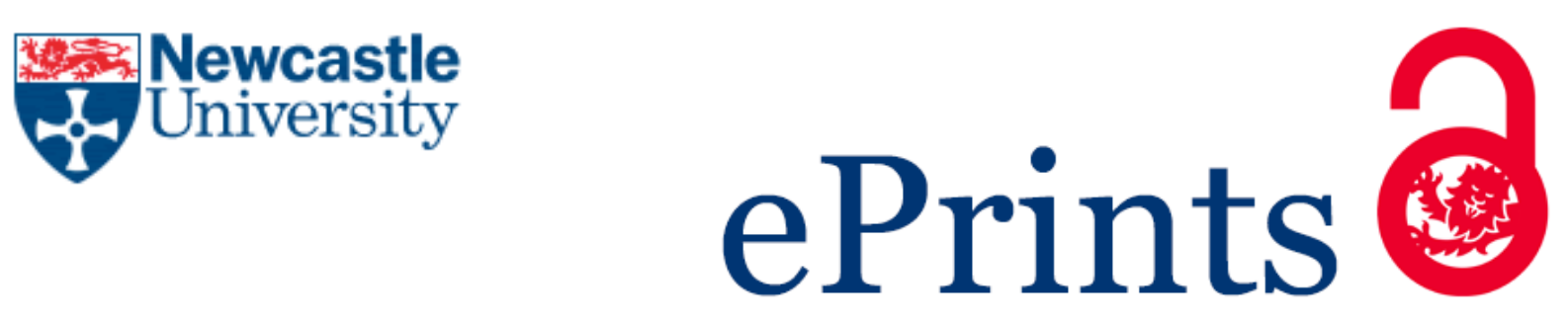

\author{
Ramezani R, Dehkhoda F, Soltan A, Degenaar P, Liu Y, Constandinou T. \\ An Optrode with Built-in Self-Diagnostic and Fracture Sensor for Cortical \\ Brain Stimulation.
}

In: 2016 IEEE Biomedical Circuits and Systems Conference (BioCAS). 2017, Shanghai: IEEE.

\title{
Copyright:
}

(C) 2016 IEEE. Personal use of this material is permitted. Permission from IEEE must be obtained for all other uses, in any current or future media, including reprinting/republishing this material for advertising or promotional purposes, creating new collective works, for resale or redistribution to servers or lists, or reuse of any copyrighted component of this work in other works

DOI link to article:

http://dx.doi.org/10.1109/BioCAS.2016.7833814

Date deposited:

$20 / 04 / 2017$ 


\section{An Optrode with built-in self-diagnostic and fracture sensor for cortical brain stimulation}

\author{
Reza Ramezani, Fahimeh Dehkhoda, Ahmed Soltan \\ and Patrick Degenaar \\ School of Electrical and Electronic Engineering \\ Newcastle University, UK \\ Email: reza.ramezani@ncl.ac.uk
}

\author{
Yan Liu and Timothy Constandinou \\ Department of Electrical and Electronic Engineering \\ Imperial College London, \\ London, UK
}

\begin{abstract}
This paper proposes a self-diagnostic subsystem for a new generation of brain implants with active electronics. The primary objective of such probes is to deliver optical pulses to optogenetic tissue and record the subsequent activity, but lifetime is currently unknown. Our proposed circuits aim to increase the safety of implanting active electronic probes into human brain tissue. Therefore, prolonging the lifetime of the implant and reducing the risks to the patient. The selfdiagnostic circuit will examine the optical emitter against any abnormality or malfunctioning. The fracture sensor examines the optrode against any rapture or insertion breakage. The optrode including our diagnostic subsystem and fracture sensor has been designed and successfully simulated at $350 \mathrm{~nm}$ AMS technology node and sent for manufacture.
\end{abstract}

Keywords-optrode; optical stimulation; epilepsy;

\section{INTRODUCTION}

Optogenetics has shown a great potential to treat brain neurological conditions such as epilepsy, Parkinson and depression [1, 2]. In this technique, neuron cells are genetically re-engineered to be photosensitive [3]. Therapy in the form of neural stimulation/control is achieved through stimulus with pulses of intense light. Closing the loop with simultaneous recording and subsequent processing can therefore be used to rectify the abnormality which created the condition.

One prerequisite to this technique is to implant a device, which can deliver pulses of intense light, inside the brain tissue $[4,5]$. This can be achieved through light guidance techniques or through implanted micro-emitters. The former creates challenges with signal multiplexing and optical alignment between guide and emitter. The latter requires devices and electronics closer to the target brain tissue. This raises the concerns around durability and biodegradation. In the worst case, should rupture occur, it would be undesirable to have DC current emitted into brain tissue. Such incidence can cause serious damage to the brain and often the damage is not recoverable [6]. The primary approach to prevent such damage is to hermetically seal the device (the process is called encapsulation) with non-conductive and biocompatible materials.
One source of concern is the micro-LEDs used in the optrode as the source of light [7, 8]. Micro-LEDs are components which will be bonded on the surface of the optrode down the needle part (so-called shaft) before the encapsulation process. Due to the heating generated by micro-LEDs, the bonding quality may degrade over time. This could potentially cause the damage to the optrode operation. Another source of concern is shaft fracture during insertion as silicon is a brittle material. Upon such incidence, the isolator can no longer be effective and DC currents may be emitted into the tissue.

In this paper, we propose a built-in self-diagnostic subsystem to examine the status of micro-LEDs and also a fracture sensor to verify the status of the optrode shaft after it is implanted.

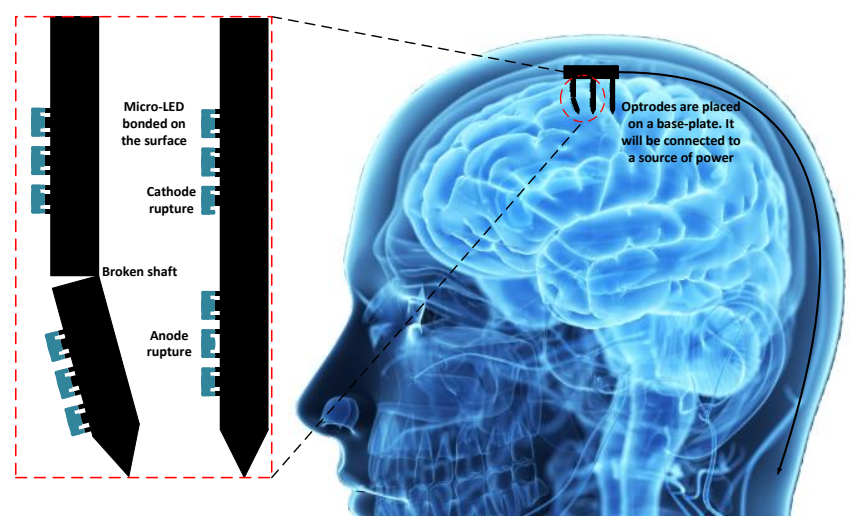

Fig. 1. The breakage can happen at the shaft of the optrode or at microLEDs bonding.

\section{OPTRODE ARCHITECTURE}

In general, optrode is utilized to perform brain cell activity recording as well as stimulation. The recording is conventionally done by placing single or multiple electrodes close to the cells. The recorded signal is then amplified and quantized to be used in the digital domain for further analysis. The results of such analysis later will be used to stimulate the brain cells. Stimulation is done by shining light to the cells with neurons cells which are genetically modified to be photosensitive. 


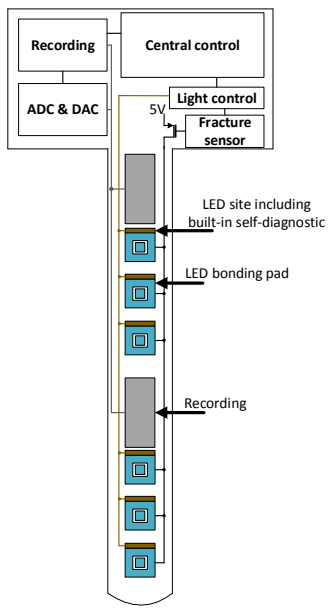

Fig. 2. Fracture sensor and built-in self-diagnostic subsystem integrated in the design of the optrode.

The designed optrode consists of following parts: (a) central control; governs the operation of the optrode such as recording, stimulation and diagnostic, (b) analogue to digital converter (ADC) and digital to analogue converter (DAC) are used in recording and diagnostic operation, (c) light control sub-circuit is used to control the intensity of the micro-LED light, (d) fracture sensor to examine the state of the shaft of the optrode.

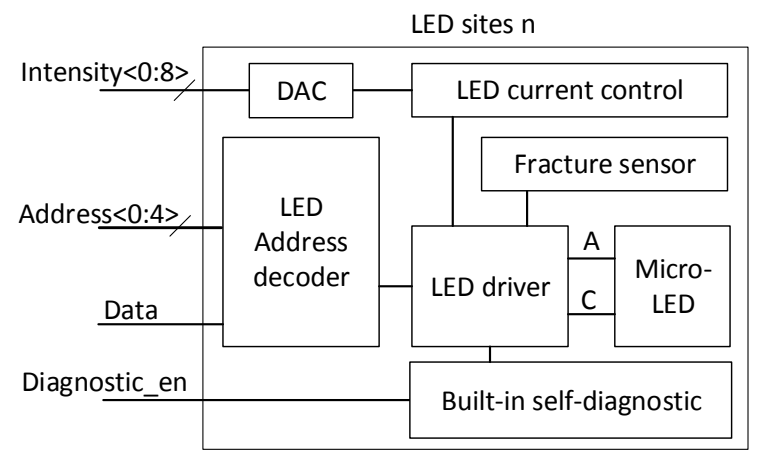

Fig. 3. The block diagram of the micro-LED sites containing sub-circuit to perform diagnostic.

\section{LED SITES}

Each probe will have a number of identical sites along its shaft for neural interfacing. Each site will contain hardware for optical stimulation (via micro-LEDs) and also circuit to perform diagnostic operation. The optical stimulation is governed by the central control unit. It uses a DAC to attain light intensity modulation. The LED current control and the LED driver convert the voltage provided by DAC into current signal and deliver it to micro-LED. The LED site will also perform diagnostic operation including micro-LED diagnostic and fracture sensor. Fig. 3 depicts the architecture of an LED site.

\section{DIAGNOSTIC SUBSYSTEM}

It is important for the optrode to evaluate any operational degradation. Non-functional components could then be switched off. This not only saves energy, but also could prevent undesirable current discharge into the tissue. Under normal operation, the current through the LED will have a diode-like behavior with applied voltage until limited by the control transistor. If however there is a significant abnormality such as an open circuit this can be seen in the voltage at that point.

The diagnostic circuit fundamentally consists of an 8-bit capacitive-based DAC and a buffer. By imparting different voltages from DAC, different currents will be passed to the micro-LED. If there is significant resistance (e.g. contact corrosion between CMOS and micro-LED) then the diodelike behavior would become more resistive. Alternatively, if there is an open circuit formed the LED I-V characteristics will be altered. Fig. 5 shows the block diagram of the proposed self-diagnostic subsystem.

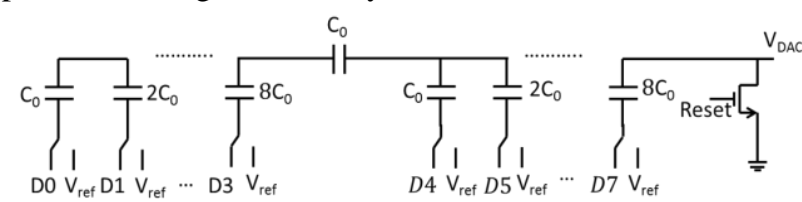

Fig. 4. Split capacitor DAC to control the LED current.

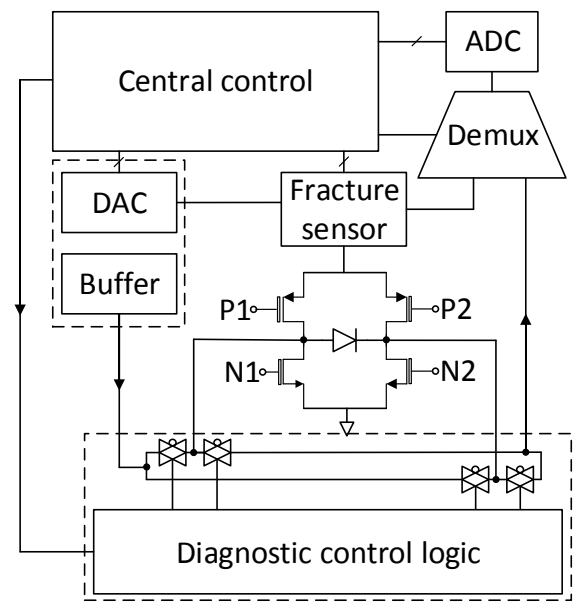

Fig. 5. Diagnostic subsystem will examine the status of the micro-LED Case1; connecting LED cathode to ground and sweeping the current of the anode while monitoring the voltage of the anode. Case 2; repeat the operation on cathode while the anode is grounded.

\section{FRACTURE SENSOR}

Fig. 6 shows the architecture of the proposed fracture sensor. The assumption is that there is part of the implantable device (the head) which is placed above the brain tissue while the shaft is placed inside the tissue. Most of the circuits are placed on the head however, the stimulator (for instance micro-LEDs) are placed on the shaft. Due to the size and shape, the possibility of breakage on the shaft is high therefore, the fracture sensor is placed on the head. At the start of the operation, the main power 
switch is off, leaves no circuit down the shaft with power to operate.

At the sensing operation, the current of $M I$ is varied by a DAC controlled by the central control unit. Simultaneously, this unit, reads the voltage at the gate of $M 2$ which reflects the current flown down the shaft. Sweeping the voltage on MI creates a unique I-V profile which reflects the status of the shaft. This profile is based on the downstream circuits being powered off. Therefore, we expect the profile to reflect a significant impedance down the shaft. This profile is captured at the calibration stage before the device is being placed in the brain tissue therefore, a baseline data can be stored. Once the device is pushed inside the tissue, the shaft might break. Therefore, the impedance of the downstream is no longer the same. The broken shaft will have a huge leakage through the tissue. This will produce drastically different I-V profile while repeating the same sensing operation. If the shaft is realized to be broken the power switch will never be switched on otherwise, it is switched on and, therefore, circuits on the shaft will start working normally.

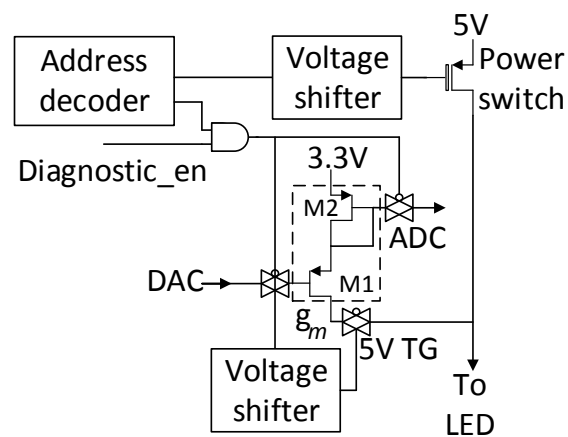

Fig. 6. In order to examine the state of the optrode, a voltage controlled current source is used. The size of the current will depends on the impedance of the shaft.

The designed optrode is expected to operate for years with next to zero potential to break down. Therefore, mitigating the probability of early degradation of any circuits is essential. One objective is to make sure $3.3 \mathrm{~V}$ transistor will never be exposed to $5 \mathrm{~V}$ signal at any moment in time. Conventional voltage shifter will not guarantee that [9]. As it is seen in Fig. 7, a voltage shifter is designed to ensure that the voltage on the $3.3 \mathrm{~V}$ transistor will never reach $5 \mathrm{~V}$.

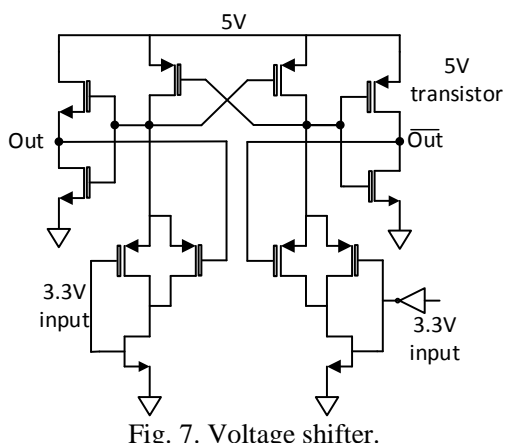

Fig. 7. Voltage shifter.

\section{SIMULATION RESULTS}

The optrode is designed and simulated at $350 \mathrm{~nm}$ technology node. This technology provides $5 \mathrm{~V}$ transistors which are required to drive micro-LEDs as well as $3.3 \mathrm{~V}$ transistors for low-power control electronics. As it is seen in Fig. 8, the micro-LED diagnostic process is done in forward and reverse bias. At forward diagnostic, the cathode of the micro-LED is grounded through N2 (Fig. 5). The central control unit sweeps the voltage of the DAC which is connected to the anode of the micro-LED. We expect that the current passing through the micro-LED to have a diodelike behavior in relationship with the DAC voltage as if the diode is in forward bias. In order to record the current of the micro-LED, the voltage on the anode is monitored using a SAR ADC. The same procedure will be followed for cathode while the anode is grounded. This will monitor the diode-like behavior as if the diode is in reverse bias. The expected results for both case are shown in Fig. 9.

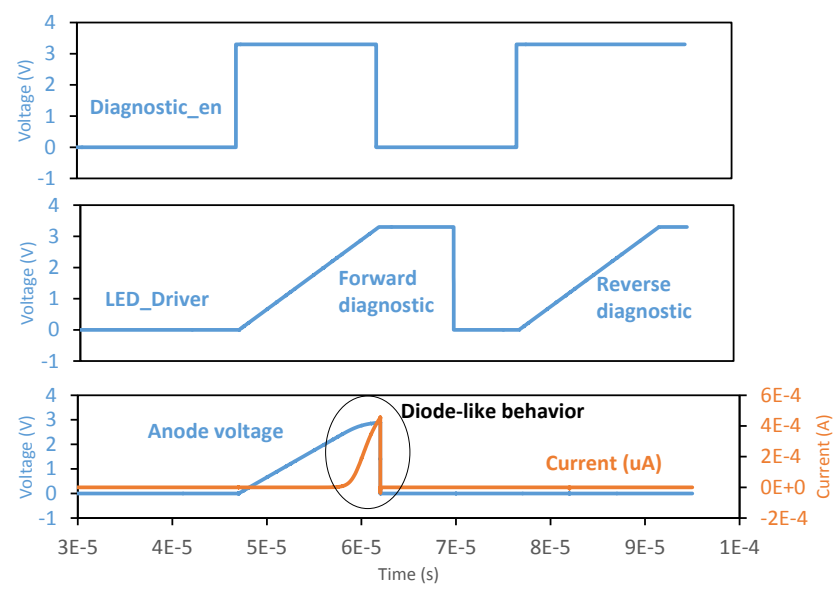

Fig. 8. The operation of the built-in self-diagnostic subsystem. Anything apart from diode-like behavior will be considered as a defect.

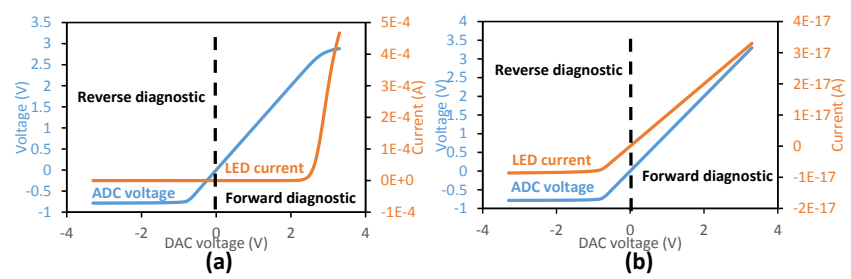

Fig. 9. Case (a) demonstrates the voltage monitored by the ADC and the current passing through the micro-LED with diode-like behavior. Case (b) represent the diagnostic while the cathode of the micro-LED is ruptured.

Fracture sensor will be configured to operate in two distinct modes: (a) sensing operation and (b) power line control. Each of these modes will have a unique address to be controlled by the central control unit. Fig. 10 depicts the operation of the fracture sensor in both modes. In sensing mode, the central control unit, along with the address, issues the sensing enable signal ("Diagnostic_en") to start the operation. The DAC voltage sweeps the voltage of $M 1$ transistor (Fig. 6) from $O V$ to $3.3 \mathrm{~V}$. As it is seen in this figure, the voltage sensed on the power line represent a 
significant impedance down the shaft. Fig. 11 shows the voltage measured at fracture sensor for different impedance down the shaft. A fractured shaft will have lower impedance and therefore, sinks bigger current.

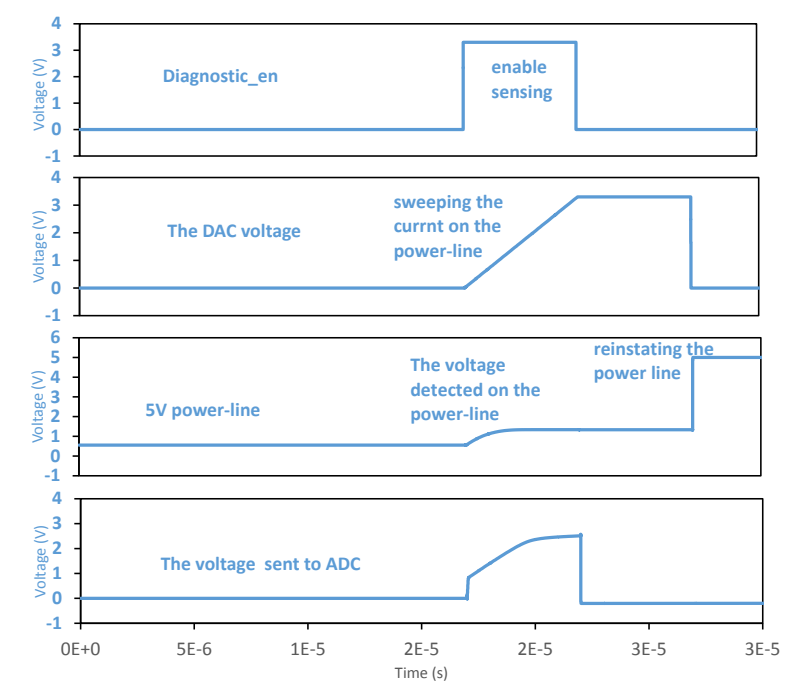

Fig. 10. The fracture sensor operation. It examines the power line and expect to detect a very high impedance as the circuits down the shaft are off. If the shaft is broken, we expect a leakage through the tissue and therefore small impedance down the shaft.

The sensing current drawn (from fracture sensor) down the shaft can be a source of concern as it can reach the tissue if the shaft is broken. In order to mitigate this risk, the $3.3 \mathrm{~V}$ supply is used instead of $5 \mathrm{~V}$ for sensing. Also, the DAC can limit the current if it exceeds the safe region.

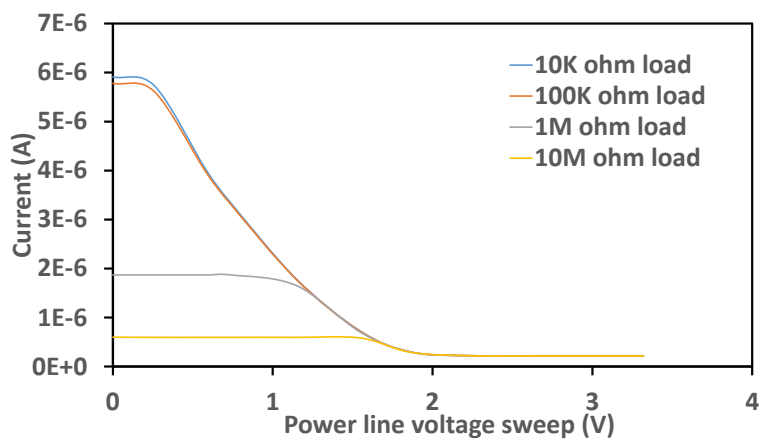

Fig. 11. Operation of fracture sensor while different impedance is loaded.

The second mode of operation of fracture sensor is to reinstate the power switch. This is done by central control unit once the sensing operation is finished. The control coming from central control unit is $3.3 \mathrm{~V}$ signal. Therefore, a voltage shifter circuit is used to fix the voltage level and drive the power switch.

\section{CONCLUSION}

In this paper, we discussed two physical defects which can endanger the operation of the implant and risk the patient's life. The bondings between the micro-LEDs and the CMOS surface of the optrode is the first source of defect. Depends upon the efficiency of the micro-LEDs, an amount of energy, is converted to light and the rest will be released as heat. The heat can potentially reduce the quality of micro-LEDs bondings over the years. This can eventually lead to complete rupture of the bondings. The proposed built-in self-diagnostic subsystem, examines the microLEDs while the device is implanted. This system scans the micro-LED I-V behavior in both forward and reverse bias. Any results which fails to depict diode-like behavior is treated as defect. The second source of defect is a broken shaft. This can cause by the force with which the optrode is positioned inside the tissue. In this paper, we propose a fracture sensor to examine the status of the shaft. In this design, a voltage control current source, is used to scan the impedance of the shaft. Any results which show significant impedance reduction is treated as defect and the device is fully powered off.

\section{ACKNOWLEDGMENT}

This work was funded by the Wellcome Trust/EPSRC Innovative Engineering for Health grant (NS/A000026/1). The authors would like to acknowledge the CANDO project (www.cando.ac.uk).

\section{REFERENCES}

[1] Patrick, D., et al., Optobionic vision-a new genetically enhanced light on retinal prosthesis. Journal of Neural Engineering, 2009. 6(3): p. 035007.

[2] Dehkhoda, F., et al. Smart optrode for neural stimulation and sensing. in SENSORS, 2015 IEEE. 2015.

[3] Nagel, G., et al., Channelrhodopsin-2, a directly light-gated cationselective membrane channel. Proceedings of the National Academy of Sciences, 2003. 100(24): p. 13940-13945.

[4] Chaudet, L., et al. Development of optics with micro-LED arrays for improved opto-electronic neural stimulation. in SPIE BiOS. 2013. International Society for Optics and Photonics.

[5] Zhao, H., et al. A CMOS-based neural implantable optrode for optogenetic stimulation and electrical recording. in Biomedical Circuits and Systems Conference (BioCAS), 2015 IEEE. 2015. IEEE.

[6] Cabrera, L.Y., E.L. Evans, and R.H. Hamilton, Ethics of the electrified mind: defining issues and perspectives on the principled use of brain stimulation in medical research and clinical care. Brain topography, 2014. 27(1): p. 33-45.

[7] Wu, F., et al., Monolithically integrated $\mu L E D s$ on silicon neural probes for high-resolution optogenetic studies in behaving animals. Neuron, 2015. 88(6): p. 1136-1148.

[8] McAlinden, N., et al., Optogenetic activation of neocortical neurons in vivo with a sapphire-based micro-scale LED probe. Frontiers in neural circuits, 2015. 9: p. 25.

[9] Osaki, Y., et al., A Low-Power Level Shifter With Logic Error Correction for Extremely Low-Voltage Digital CMOS LSIs. IEEE Journal of Solid-State Circuits, 2012. 47(7): p. 1776-1783. 